\title{
Creasensor: SIMPLE technology for creatinine detection in plasma
}

2
Francesco Dal Dosso, Deborah Decrop, Elena Pérez-Ruiz, Devin Daems, Hannah Agten, Osamah Al-Ghezi, Olivier Bollen, Jolien Breukers, Florian De Rop, Maria Katsafadou, Jens Lepoudre, Linye Lyu, Pieter Piron, Robbe Saesen, Shoera Sels, Rani Soenen, Ellen Staljanssens, Jehan Taraporewalla, Tadej Kokalj, Dragana Spasic, and Jeroen Lammertyn* BIOSYST-MeBioS, KU Leuven, Leuven, Belgium

*jeroen.lammertyn@kuleuven.be

\section{Abstract}

The lab-on-a-chip (LOC) field has witnessed an excess of new technology concepts, especially for the point-of-care (POC) applications. However, only few concepts reached the POC market often because of challenging integration with pumping and detection systems as well as with complex biological assays. Recently, a new technology termed SIMPLE was introduced as a promising POC platform due to its features of being self-powered, autonomous in liquid manipulations, cost-effective and amenable to mass production. In this paper, we improved the SIMPLE design and fabrication and demonstrated for the first time that the SIMPLE platform can be successfully integrated with biological assays by quantifying creatinine, biomarker for chronic kidney disease, in plasma samples. To validate the robustness of the SIMPLE technology, we integrated a SIMPLE-based microfluidic cartridge with colorimetric read-out system into the benchtop Creasensor. This allowed us to perform on-field validation of the Creasensor in a single-blind study with 16 plasma samples, showing excellent agreement between measured and spiked creatinine concentrations (ICC: 0.97). Moreover, the range of clinically relevant concentrations $(0.76-20 \mathrm{mg} / \mathrm{dL})$, the sample volume $(5 \mu \mathrm{L})$ and time-to-result of only $5 \mathrm{~min}$ matched the Creasensor performance with both lab based and POC benchmark technologies. This study demonstrated for the first time outstanding robustness of the SIMPLE in supporting the implementation of biological assays. The SIMPLE flexibility in liquid manipulation and compatibility with different sample matrices opens up numerous opportunities for implementing more complex assays and expanding its POC applications portfolio.

Keywords: Lab-on-a-chip; point-of-care; self-powered microfluidic pump; creatinine; chronic kidney disease

\section{Introduction}

Since their introduction more than twenty years ago, lab-on-a-chip (LOC) devices generated high interest and expectations within the microfluidics community and beyond [1-3]. In particular, their intrinsic properties such as miniaturization, integration of different laboratory processes, rapid time-to-result (TTR) and low cost fabrication, envisaged their bright future for point-of-care (POC) testing [3,4]. However, despite the initial excitement, the LOC devices that found their way to the POC market continue to be greatly outnumbered by those remaining in the research environment. This phenomenon can be mostly explained with two arguments. First, researchers have been often focusing on increasing the versatility and thus also the 
complexity of the microfluidic chips without simultaneously working on their integration with pumps and readout systems. As a consequence, lab-on-a-chip became chip-in-a-lab and resulted in small microfluidic chips needing bulky instrumentation to operate, which is suitable for research purposes but not for POC applications [3,5]. Second, the trend in the LOC community for many years was to develop new technologies rather than to integrate the existing ones with biological applications and make them more robust and user-friendly for non-technical users $[6,7]$.

Number of autonomous and self-powered microfluidic approaches tried to address the integration and portability aspects. For instance, systems based on capillary forces of intricate microstructures were introduced as capable of drawing liquids into a microfluidic network and performing complicated multi-step assays [8]. However, these systems required hydrophilization of the surfaces to obtain the capillary effect increasing the complexity and cost of the fabrication. Other platforms were based on the so called "degas-driven flow" concept [913], which takes advantage of the inherent high porosity and air solubility of PDMS. However, this approach was limited to permeable materials while the control over flow rate and timing was challenging. A third promising approach was paper-based microfluidics where paper is exploited as pumping element relying on capillary action to move liquids. The paper strip was often patterned with different reagents at different locations with the final goal to perform multi-step tests [14-17]. Although promising, the performance of the bio-assay integrated on the paper-based microfluidic platforms is often hampered by the irreproducible fluid control, the narrow range of flow rates and the interaction of the sample with the matrix.

More recently, a new self-powered pumping concept has been introduced, being the Self-powered Imbibing Microfluidic Pump by Liquid Encapsulation (SIMPLE) [18] which works in pulling mode. Although the potential of this technology for POC applications is enormous due to its versatility in terms of flow rate and liquid volume, autonomy in liquid manipulation, low cost, ease-of-fabrication and suitability for mass-replication it has not been combined so far with any biological application. Therefore, in this paper we show for the first time that the SIMPLE platform can be effectively integrated with a model bioassay, i.e. an enzymatic assay for quantification of creatinine. Creatinine is the golden standard biomarker for chronic kidney disease (CKD) and it is sufficient for diagnosing this disease and monitoring of its progress [1921], which is a medical condition associated with the gradual loss of kidney function, leading to kidney transplantation in the acute cases [22]. Moreover, starting from the previously published SIMPLE concept, in this paper we improve the fabrication and design process. To validate the robustness of the SIMPLE technology, we further combine SIMPLE-based microfluidic cartridge (Creacard) with colorimetric read-out system into the fully integrated Creasensor device. Using Creasensor, we quantify creatinine directly in a plasma matrix within the entire clinically relevant concentration range and we validate on-field the performance of the device in a singleblind study. We finally benchmark the performance of the newly developed SIMPLE biosensor with the well-established laboratory-based and POC platforms for creatinine diagnostics.

\section{Materials and methods}

\subsection{Reagents and materials}

All buffer reagents and creatinine anhydrous (98\% purity) were purchased from The Merck group - Sigma Aldrich (Belgium), as well as creatinine standard solutions, creatinine assay buffer, creatinine probe, creatininase, creatinase and creatinine enzyme mix, which were 
obtained from the Creatinine Assay kit (MAK080). Bovine plasma heparin NA was supplied by Tebu-Bio (Belgium). All solutions were prepared using deionized water purified with a Milli-Q 50 ultrapure water system (Millipore, Belgium). Double-sided pressure sensitive adhesive (PSA) tape (200MP-7945MP) was provided by 3M (USA). PVC transparent foils of two different thicknesses (180 and $300 \mu \mathrm{m})$ were utilized. Whatman qualitative filter paper grade 6 was acquired from The Merck group - Sigma Aldrich (Belgium). The following components were used for the electronic circuits: TLLG440 green LEDs (Vishay, USA), TMP36GT9Z temperature sensor (Analog Devices, USA), VNP20N07 mosfet (ST, USA), Arduino UNO (Arduino, Italy), TSL257-LF light-voltage converter (Austria Micro System, Austria), 5V heating pad (SparkFun Electronics, USA), power bank 2 USB Ports 10000 mAh (TP-Link, China), Gameduino 2 (Adafruit, USA) and resistors, wires and two-ways switches from AITEC (Belgium). FB570-10 bandpass filter was acquired from Thorelabs (USA). Black PMMA plates ( $3 \mathrm{~mm}$ thick) were cut with a speedy 100R laser cutter from Trotec (Austria).

2.2. Fabrication of the microfluidic cartridge, colorimetric read-out and integrated Creasensor device

The microfluidic cartridge, Creacard, was fabricated according to the low-cost and rapid prototyping method presented in Yuen et al. [23], and Dal Dosso et al. [24]. The microfluidic network was cut in different PSA layers by using a digital tabletop craft cutter (Maxx Air 24", KNK, USA). The Creacard was divided in two units: a pump, termed the SIMPLE unit [18] and the detection unit. These two units were connected to each other via the connection hole (Figure 1A).

In the SIMPLE unit, the PSA layer was sandwiched between a bottom $(300 \mu \mathrm{m})$ and top $(180 \mu \mathrm{m})$ PVC layer. The top layer was designed with connection, prefilling and vent holes. A porous material, in this case filter paper (Grade 6, Whatman) shaped with the digital craft cutter, was inserted into its chamber during the assembly (Figure 1A).

The detection unit was fabricated with four PSA layers, where the inlet channel was connected to the top part of the detection unit, while the outlet channel, coupled to the SIMPLE unit via the connection hole, was positioned at the bottom of the detection unit (Figure 1A). The chamber of the detection unit was designed with a height of $508 \mu \mathrm{m}$ (Figure 1B, side view). After fabrication, the working liquid was prefilled through the prefilling hole bringing the working liquid close to but not in contact with the porous material, as previously described [18], which was then sealed. In this case, blue food dye diluted in distilled water (1:100) was used as working liquid. A side and a top view of the final assembled and prefilled Creacard is depicted in Figure 1B. 

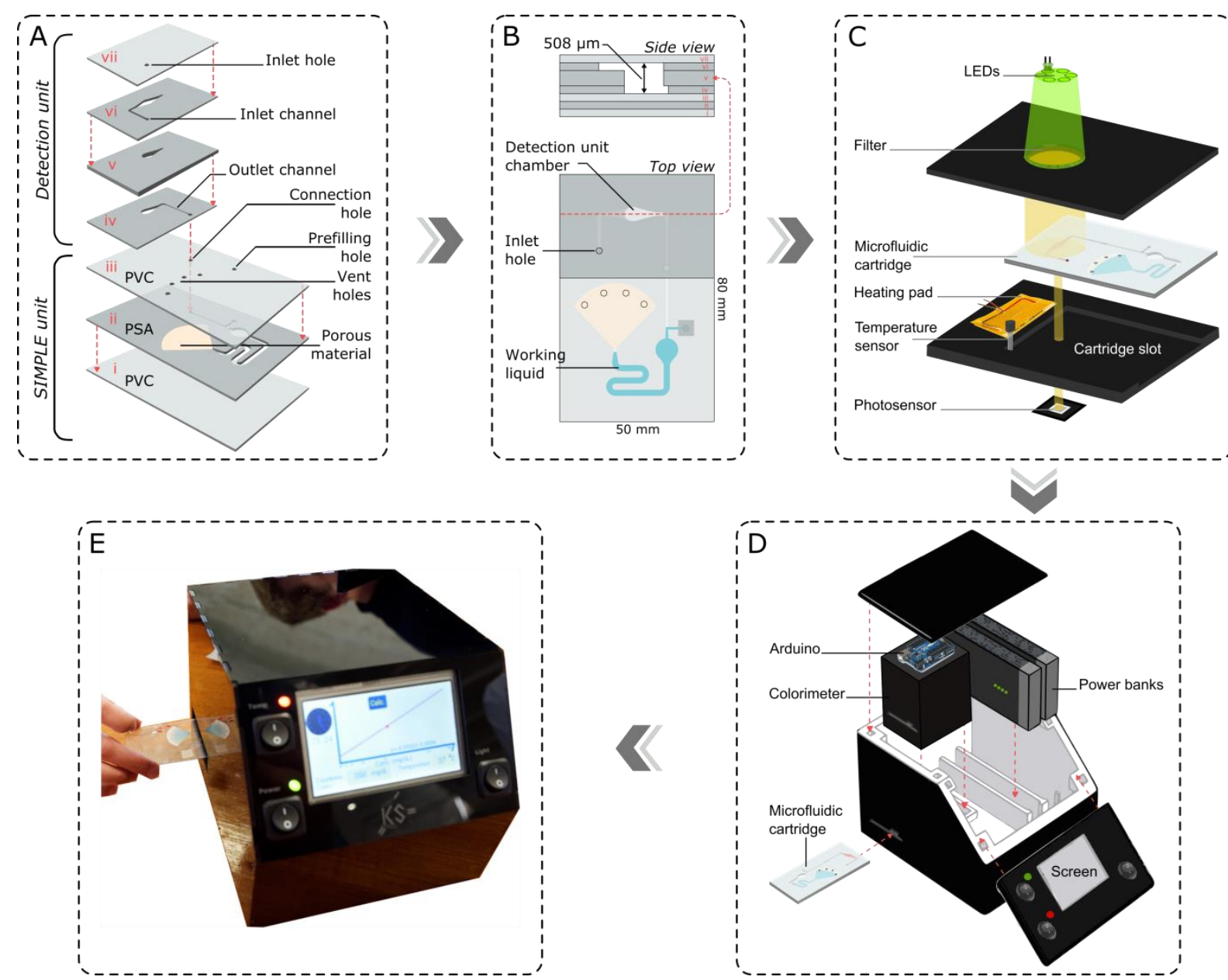

Figure 1 Overview of the Creacard (microfluidic cartridge), colorimeter and integrated Creasensor. A) Fabrication of the Creacard comprising the SIMPLE unit and the detection unit. The assembly sequence of the cartridge is presented with red arrows and numbers. B) Top view of the assembled and prefilled Creacard and side view of the detection unit (chamber height $=508 \mu \mathrm{m}$ ). C) Exploded view of the main components of the colorimeter. D) Exploded view of the Creasensor with the colorimeter, an Arduino microcontroller, power banks and a screen. E) Photo of the final Creasensor device in use.

The Creasensor was designed and built to provide a robust and fast read-out for creatinine measurements using a colorimeter (Figure 1C). Here, the light of six green LEDs was filtered by a band-pass filter to select the proper wavelength $(570 \mathrm{~nm})$ that matched the bioassay requirements. After passing through the detection zone of the microfluidic cartridge, the filtered light reached the photosensor. The detection zone was specifically designed to be higher than the rest of the cartridge channels in order to ensure a sufficient path length for the colorimetric read-out. In fact, longer path lengths, in agreement with the Beer-Lambert law, are preferred to measure absorbance of a sample, since it is intimately dependent on the path length. The different layers of the colorimeter were made with black PMMA plates and inserted in a compact black box to force the light going only through the desired path and to prevent any external light affecting the measurement. The colorimeter box had only one opening for inserting the cartridge and, integrated on the cartridge slot plate, a heating pad and a temperature sensor. The heating pad and temperature sensor were integrated in the Creasensor to ensure environment with a controlled temperature. In particular, the heating pad was used for generating heat to reach $37^{\circ} \mathrm{C}$ in the colorimeter chamber, while the temperature sensor, controlled by the Arduino microcontroller, was used for real-time monitoring of the 
placed in the final device with all the other components: a microcontroller (Arduino Uno), which controlled both the photosensor and temperature sensor and processed the data, an LCD screen where the results were displayed and two power banks that provided enough power to the components (Figure 1D). Three switches were placed on the front panel to easily switch on/off the light source, the microcontroller and the heating element. Detailed electronic schemes and connections are presented in supplementary information (Figure S1, Figure S2, Figure S3). The final assembled Creasensor during operation is presented in Figure 1E.

\subsection{Creatinine enzymatic assay in buffer for POC application}

Creatinine samples were prepared by diluting a $100 \mathrm{mM}$ creatinine standard in $50 \mu \mathrm{L}$ of creatinine assay buffer (previously equilibrated to RT) to obtain different final concentrations of creatinine ranging from 0 to $2.26 \mathrm{mg} / \mathrm{dL}(0,0.45,0.90,1.35,1.81,2.26 \mathrm{mg} / \mathrm{dL})$. These solutions were mixed with $50 \mu \mathrm{L}$ of creatinine master mix in a 96-well plate (Costar 3590, Corning, USA). The master mix was prepared by mixing $2 \mu \mathrm{L}$ of creatinine probe (previously heated at $37^{\circ} \mathrm{C}$ for $3 \mathrm{~min}$ in water bath), $2 \mu \mathrm{L}$ of creatinase, $2 \mu \mathrm{L}$ of creatininase, $2 \mu \mathrm{L}$ of enzyme mix and $42 \mu \mathrm{L}$ of creatinine assay buffer. After thorough mixing, the absorbance was measured using a microplate reader plate (SpectraMax $\AA$ M Series Multi-Mode microplate reader, Molecular devices, USA) at $570 \mathrm{~nm}$ every $5 \mathrm{~min}$ for a period of $60 \mathrm{~min}$, while incubating at $37^{\circ} \mathrm{C}$. More details on the enzymatic cascade are presented in Section 3 of the Supplementary Information. To optimize the assay for integration with Creasensor (i.e. POC application), a smaller sample volume (i.e. $20 \mu \mathrm{L}$ ) was spiked with creatinine and mixed with $20 \mu \mathrm{L}$ of creatinine master mix (by proportionally scaling down all assay components). The obtained $40 \mu \mathrm{L}$ of sample was used for loading the Creacard.

\subsection{Optimized creatinine assay on the Creasensor}

In order to calibrate the Creasensor, the bioassay was first optimized in bulk with plasma samples, as described in Section 4 of the Supplementary information, followed by implementation on the Creacard and measuring with the colorimeter. Six different concentrations of creatinine were spiked in $5 \mu \mathrm{L}$ of plasma and diluted in $35 \mu \mathrm{L}$ of creatinine master mix (1:8 dilution factor) to obtain final concentrations of 1, 2, 4, 8, 16 and $20 \mathrm{mg} / \mathrm{dL}$. For each concentration, the total obtained volume of $40 \mu \mathrm{L}$ was loaded on the Creacard. The cartridge was subsequently activated by a fingertip press on the activation zone and inserted in the colorimeter after $90 \mathrm{~s}$ (allowing the sample to completely fill the detection zone) to start the measurement. The measurement was stopped 5 min after mixing of the sample and the master mix. A linear regression was performed on the real-time photosensor signal to obtain the slope of the signal curve, which is proportional to the creatinine concentration. Detailed signal processing procedure is presented in Section 5 of the Supplementary Information.

\subsection{On-field validation of the Creasensor device}

The Creasensor was validated in a single-blind study with 16 concentrations of creatinine in the range of $0-20 \mathrm{mg} / \mathrm{dL}$ spiked in blood plasma. The amount of creatinine in these biological samples was quantified following the procedure described in Section 2.4. When the unknown samples were measured with the Creasensor, the results were compared with the respective creatinine concentrations and the intraclass correlation coefficient (ICC) [25] was calculated 
with the statistical package $\mathrm{R}$ (version 2.11.1, The R foundation for Statistical Computing, Austria).

\section{Results and discussion}

3.1. Development of the microfluidic cartridge and Creasensor device

To develop a fully integrated SIMPLE-based biosensor for creatinine quantification, two main parts were designed and built in this work: (i) the Creacard (Figure 2), a microfluidic cartridge based on the SIMPLE concept [18] and (ii) a portable colorimetric read-out system. The main role of the SIMPLE-based cartridge was to efficiently pull the sample into the microfluidic network in order to obtain a standardized filling of the detection zone. The cartridge was prefilled with working liquid (blue) through the prefilling hole, while a droplet of sample (pink) was deposited on the inlet hole (Initiation). The working liquid can be seen as the "fuel" of the pump (i.e. porous material) that never gets in contact with the sample. The pump was then simply activated by a fingertip press on the activation zone, bringing the working liquid in contact with the porous material (Activation). The absorption of the working liquid by the porous material caused a reduced pressure in the inlet channel, pulling the sample into the microfluidic network (Operation). The pump was designed to stop when the detection zone was completely filled due to complete absorption of working liquid (Termination). The design of the microfluidic network was optimized to ensure a consistent, complete filling of the detection zone without bubble formation. The total pump operation time was about $90 \mathrm{~s}$, providing fast liquid manipulation.

The colorimetric read-out system of the Creasensor was developed as described in Section 2.2. Before integrating the optimized bioassay into the Creasensor (see Section 3.4), the stability of the photosensor signal and of the temperature over time were tested. Hence, the baseline signal of the photosensor (i.e. when no cartridge was inserted) was recorded over 45 min and analyzed. A negligible variation was observed $(3.050 \pm 0.007 \mathrm{~V})$, proving the robustness of the read-out system (Figure S4). To check the temperature stability, the Arduino microcontroller was used to collect the temperature measured by the temperature sensor, compare it to the desired temperature and switch on/off the heating pad to reach and keep this temperature (Figure S3). This system was able not only to increase the temperature from RT to the set temperature (around $37^{\circ} \mathrm{C}$ ) in about $30 \mathrm{~min}$, but also to keep it constant over time thanks to the feedback loop integrated in the code. It is important to note that the assay was performed at $37{ }^{\circ} \mathrm{C}$ based on recommendations of the kit manufacturer as well as on the observed effect of the temperature on the assay performance (Figure S5). 

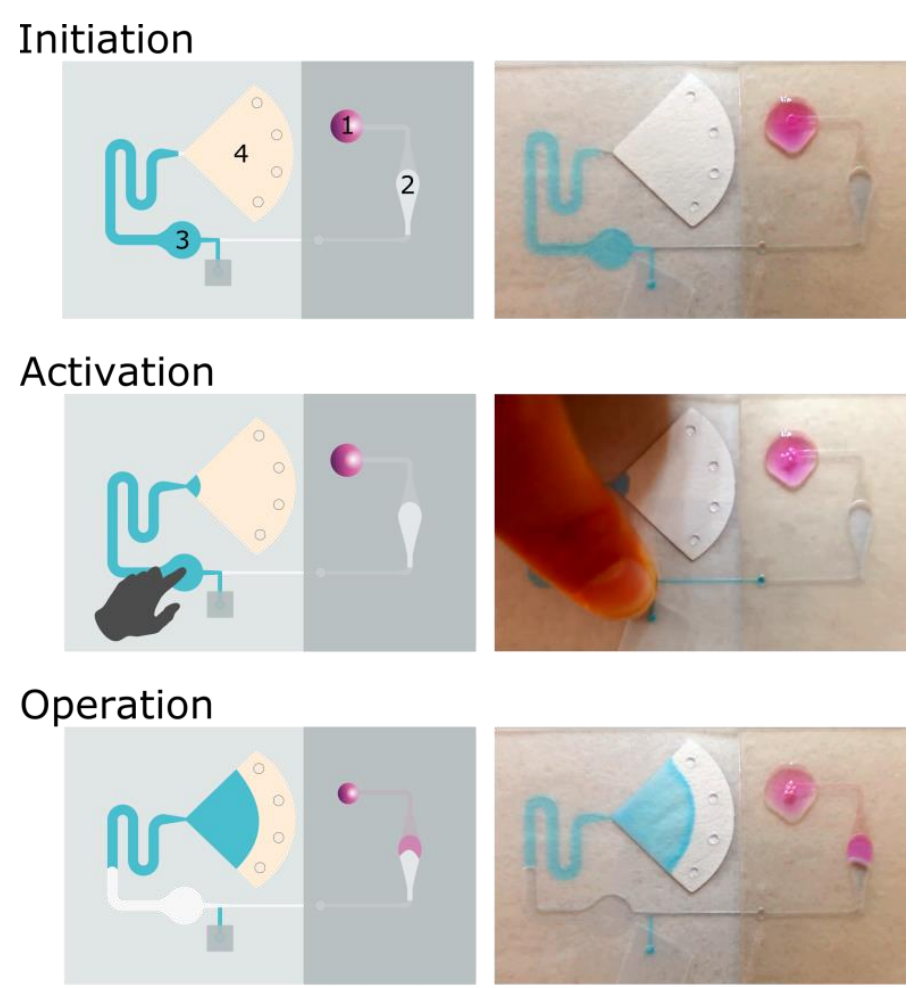

\section{Termination}
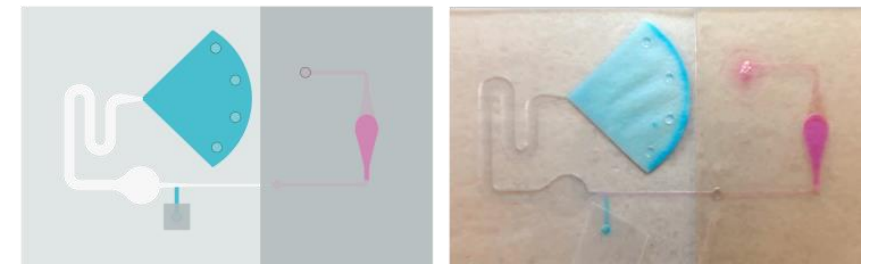

Figure 2 Microfluidic cartridge operation. The pump consists of an 1) inlet hole with sample (pink), 2) detection zone, 3) working liquid chamber with working liquid (blue) and 4) a chamber with porous material. Schematic representation of the pumping concept is shown on the left side, while the snapshot photos of the actual experiment are shown on the right side.

3.2. Optimization of creatinine enzymatic assay in buffer for POC application

As a first step towards integration in the Creasensor, the commercially-available enzymatic assay for creatinine (The Merck group - Sigma Aldrich, Belgium) was optimized in buffer using microtiter plates for the following two aspects: sample volume (i.e. volume of the buffer needed for spiking creatinine) and TTR. The aim was to keep the sample volume between 5 and $20 \mu \mathrm{L}$, as this would correspond to the typical volume obtained from finger prick sampling [26], and the TTR lower than $15 \mathrm{~min}$, since both conditions are preferred for POC applications [27].

The effect of different sample volumes on the assay performance was investigated by testing $50 \mu \mathrm{L}$, which is the volume suggested by the manufacturer of commercially-available creatinine kit, and $20 \mu \mathrm{L}$. The samples were prepared by spiking in buffer six different creatinine concentrations to reach final concentrations of $0,0.45,0.90,1.35,1.81,2.26 \mathrm{mg} / \mathrm{dL}$. These concentrations overlap with the typical human reference range of creatinine in blood, namely between 0.5 and $1.2 \mathrm{mg} / \mathrm{dL}$ for healthy people [28], but easily exceeding $6 \mathrm{mg} / \mathrm{dL}$ in patients affected by CKD [29]. When the absorbance was measured at $37^{\circ} \mathrm{C}$ every $5 \mathrm{~min}$ for each concentration, obtained kinetic curves revealed that discrimination between all the concentrations was possible already at $5 \mathrm{~min}$ incubation irrespective of the sample volume (Figure S6). This suggested that the readout, normally done after $60 \mathrm{~min}$ incubation of the 
samples with the creatinine master mix for the commercial assay, could be reduced to 5 min. Hence, a calibration curve for each sample volume was generated plotting the absorbance measured after only $5 \mathrm{~min}$ in function of the creatinine concentrations (Figure 3A). The response curve was around $73 \%$ less steep for $20 \mu \mathrm{L}$ samples (black curve) compared to the one built using $50 \mu \mathrm{L}$ sample (blue curve) in agreement with the Beer-Lambert law. However, both calculated LODs, 0.006 and $0.059 \mathrm{mg} / \mathrm{dL}$ respectively for $50 \mu \mathrm{L}$ and $20 \mu \mathrm{L}$ sample volume, were well below creatinine blood levels in kidney patients. The LOD was calculated as $\mathrm{x}_{0}+3 \times \mathrm{s}_{0}$, where $\mathrm{x}_{0}$ is the average response for $0 \mathrm{mg} / \mathrm{dL}$ creatinine and $\mathrm{s}_{0}$ is the mean standard deviation for $\mathrm{x}_{0}$.

From these results, and taking into account that lower sample volumes are preferred for POC devices, $20 \mu \mathrm{L}$ was chosen for the following experiments, resulting in a total reaction volume of only $40 \mu \mathrm{L}$ in buffer matrix once mixed with creatinine master mix. Additionally, these measurements proved that creatinine can be reliably measured in buffer samples after only 5 min incubation using this enzymatic reaction, thus reducing the TTR more than 90\% A

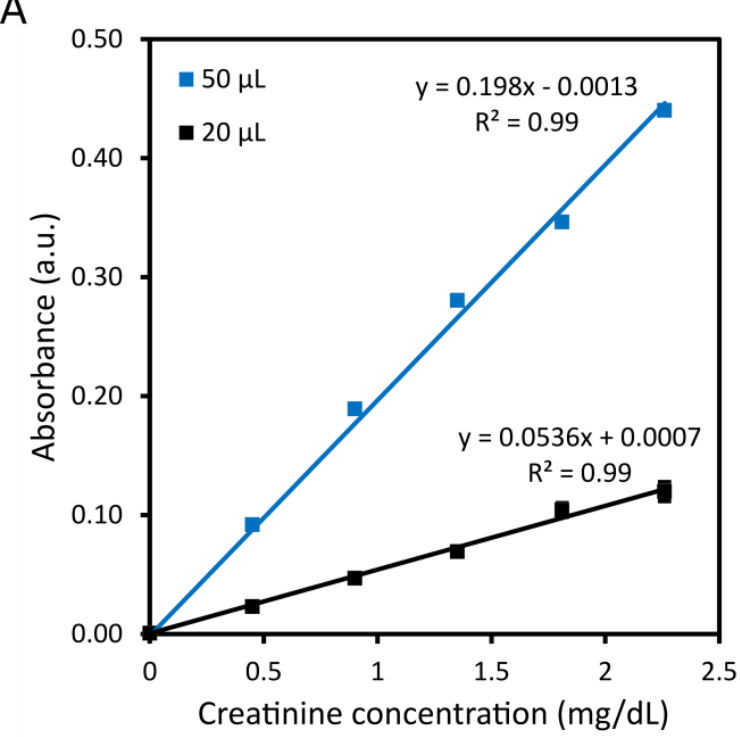

B

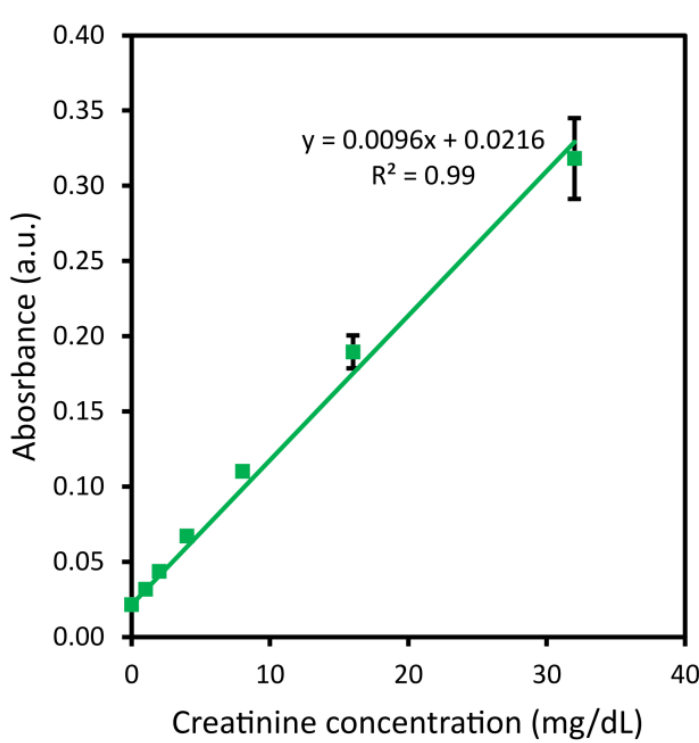

Figure 3 Creatinine enzymatic assay optimization for POC application. A) Plots representing the measured absorbance as a function of the creatinine concentration $(0,0.45,0.90,1.35,1.81,2.26 \mathrm{mg} / \mathrm{dL})$ after 5 min incubation time at $37{ }^{\circ} \mathrm{C}$ for two different assay conditions: $50 \mu \mathrm{L}$ (blue) and $20 \mu \mathrm{L}$ (black) sample volume in buffer. Linear fits are added $\left(R^{2}=0.99\right.$ for both curves). Error bars represent the standard deviation based on three repetitions (error bars are the same size or smaller than the symbols). B) Quantification of creatinine in a plasma matrix (1:8 dilution) after 5 min incubation time. The plot represents the measured absorbance as a function of the creatinine concentration $(0,1,2,4,8,16,32$ $\mathrm{mg} / \mathrm{dL}$ ). A linear fit is added for which $\mathrm{R}^{2}=0.99$. Error bars represent the standard deviation based on three repetitions.

\subsection{Optimization of creatinine detection in a plasma matrix}

Because creatinine levels are commonly measured in urine or plasma, the performance of the optimized enzymatic assay was further tested in a plasma matrix. To do so, the dilution factor of the samples was investigated to minimize the interference from substances that might be present in clinical samples and can either increase the background or suppress the specific signal. As presented in Figure S7, 1:8 dilution factor led to the best signal-to-noise ratio when using only $5 \mu \mathrm{L}$ of plasma sample and was selected for building a calibration curve with different creatinine concentrations. 
Although the commercial enzymatic assay is designed to measure creatinine values in a narrow range (from 0 and $2.26 \mathrm{mg} / \mathrm{dL}$ ), the desired POC test should be able to measure creatinine in a wider range, and hence for this experiment creatinine samples were measured from 0 to $32 \mathrm{mg} / \mathrm{dL}$ using the protocol summarized in Section 4 of the Supplementary information. The obtained calibration curve is depicted in Figure 3B. A linear trend was observed over the tested concentration range leading to a calculated LOD of $0.68 \mathrm{mg} / \mathrm{dL}$. When comparing these results with the ones obtained following the protocol provided in the commercial kit (blue curve, Figure 3A), it is clear that the standard assay was successfully adapted to be implemented in a POC platform by (i) significantly reducing the TTR ( 60 min to 5 min), (ii) minimizing the sample volume (50 $\mu \mathrm{L}$ in buffer to $5 \mu \mathrm{L}$ in plasma samples) and (iii) using a clinically relevant matrix (blood plasma instead of buffer). Although the obtained LOD in plasma was higher compared to the one in buffer, it still remained within the relevant clinical range of creatinine concentrations in blood. The obtained increase in LOD value can be explained by the multiple sources of interferences present in the biological matrix, which can affect the sensitivity of the measurement. Additionally, the dynamic detection range of the assay was increased to cover a much wider concentration range $(0-32 \mathrm{mg} / \mathrm{dL}$ compared to $0-2.26$ $\mathrm{mg} / \mathrm{dL})$.

\subsection{Integration of the POC creatinine assay with the Creasensor}

300

301

302

303

304

305

306

307

308

309

310

311

312

313

314

315

316

317

318

319

320

321

322

323

324

Next, the optimized bioassay was integrated with the Creacard. For this, the Creasensor was calibrated using seven known concentrations of creatinine spiked in only $5 \mu \mathrm{L}$ of plasma samples with final concentrations covering the clinically relevant detection range for CKD patients $(0,1,2,4,8,16,20 \mathrm{mg} / \mathrm{dL})$. The samples were prepared as presented in Section 2.4. Because Creacard is designed and fabricated to be disposable (mainly to avoid cross contamination between different samples), every creatinine sample was measured using different cartridge.

As already mentioned, an important aspect of the developed read-out system was the signal processing, which is explained in detail in the supplementary information (Figure S8). Instead of using the endpoint value, a slope analysis of the enzymatic reaction curve was adopted. This approach was not affected by the errors caused by small temperature fluctuation and inter-variability in cartridge fabrication, providing a more stable and reliable read-out signal.

The calibration curve generated with this approach showed low variability (CV $<10 \%$ on average) and high linearity $\left(\mathrm{R}^{2}=0.99\right)$ over the whole tested range (grey squares in Figure 4). The Creasensor was able to discriminate between all the different concentrations tested, reaching an LOD of $0.76 \mathrm{mg} / \mathrm{dL}$. This value was comparable to the one obtained with the same assay performed in bulk (i.e. $0.68 \mathrm{mg} / \mathrm{dL}$, Figure 3B), demonstrating the robustness and sensitivity of the Creasensor. Finally, the calibration curve was validated with 5 additional samples (red squares in Figure 4). To do that, after the measurement, the creatinine concentration of these samples was back-calculated using this calibration curve. The estimated values matched the real creatinine concentrations spiked in those samples.

This was the first time a clinically relevant bioassay was implemented on the SIMPLE microfluidic cartridge and the results were comparable to the one obtained in bulk, proving that the SIMPLE concept can be effectively applied in biosensor applications. 


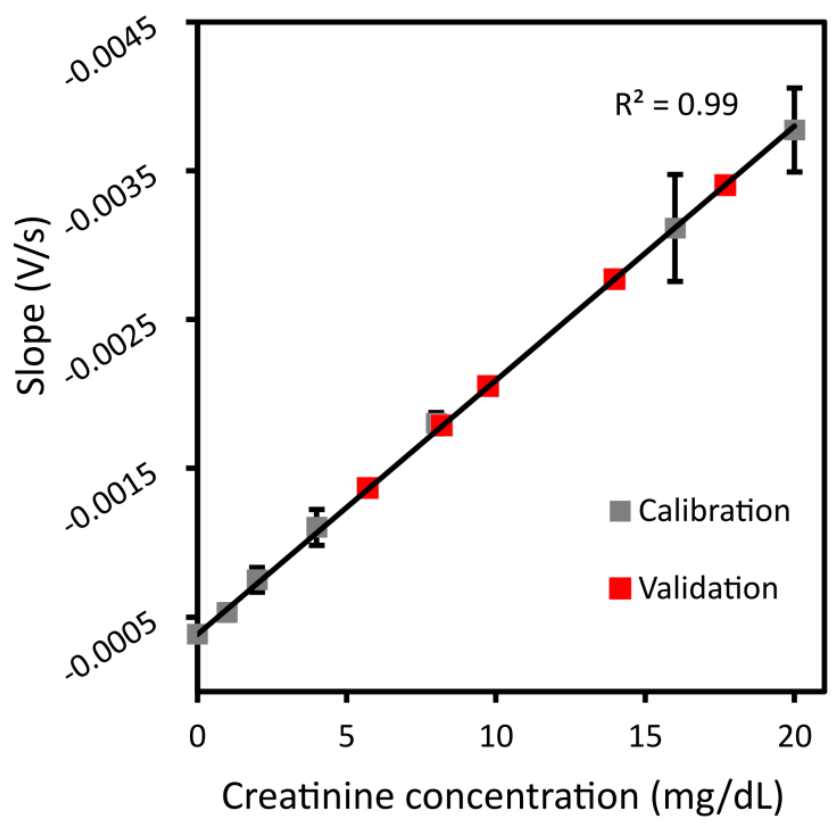

Figure 4 Calibration curve of the Creasensor in a plasma matrix (8 fold diluted) after 5 min incubation time of the samples. The plot represents the measured slope of the photosensor signal curve as a function of the spiked creatinine concentration $(0,1,2,4,8,16,20 \mathrm{mg} / \mathrm{dL})$ (grey squares). A linear fit is added for which $\mathrm{R}^{2}=0.99$. Error bars represent the standard deviation based on five repetitions. The calibration curve was validated with 5 extra samples (red squares).

3.5. On-field validation of the Creasensor device

332 The Creasensor was further validated on-field by measuring 16 plasma samples ( 8 different concentrations measured in duplicate) in a single-blind study. The samples were processed with a minimal sample preparation procedure by simply diluting $5 \mu \mathrm{L}$ of the unknown sample with the $35 \mu \mathrm{L}$ of creatinine master mix (Section 2.4), followed by loading the $40 \mu \mathrm{L}$ mixture onto the Creacard. Subsequently, the creatinine concentrations in the spiked plasma samples were determined using the calibration curve shown in Figure S9. After the measurement, the obtained concentration values were compared with the real spiked creatinine concentrations. The comparison between the creatinine concentration values measured with the Creasensor and the actual values showed a high correlation as indicated by the ICC of 0.97 , which describes how strongly the measured values resemble the reference ones (Figure 5). Moreover, for each of the 8 concentrations measured, 2 independent samples showed similar corresponding creatinine values, except for the highest concentration. These results, together with the fact that all 16 samples were measured consecutively in 3 hours, proved the robustness of the Creasensor device. 


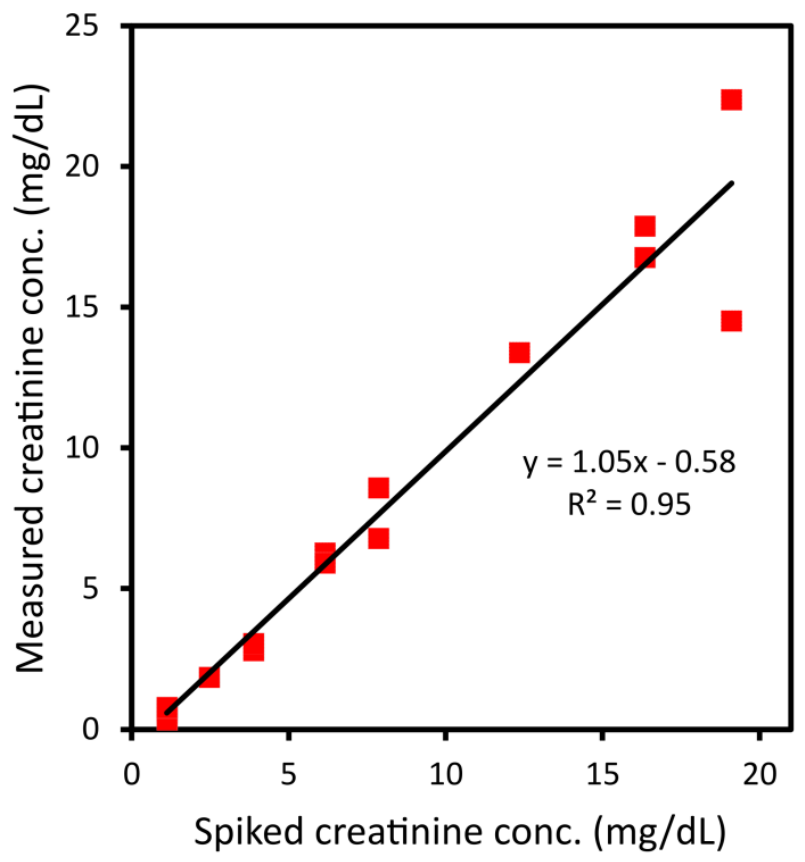

346

347

348

349

Figure 5 Validation of the Creasensor in a single-blind study. Measured creatinine concentrations of 16 samples (8 different creatinine concentrations within a physiological range, $0-19 \mathrm{mg} / \mathrm{dL}$ ) were compared to the spiked creatinine concentrations, showing linear correlation $\left(\mathrm{R}^{2}=0.95\right)$.

Over the years, other strategies for measuring creatinine have been also investigated in different research group, such as electrochemical [30,31] and optical sensors [32]. However, none of these have been adopted until now in routine laboratories mainly due to their lack of sensitivity and specificity, the partially relevant biological matrix used (i.e. urine) or their dependence on large and complex equipment. If compared with the standard creatinine detection methods, the developed Creasensor performed similarly or better in all the tested analytical parameters (see Table 1), demonstrating the strengths of the SIMPLE technology and Creasensor device. Moreover, it is important to notice that the cartridges of the standard creatinine detection methods reported in Table 1 refer to those that can measure only creatinine.

Table 1: Comparison between different commercially available creatinine biosensors and the Creasensor (adapted from $[33,34]$ ). The two most common approaches for clinical determination of creatinine are shown in the first two columns: the Jaffé method (considered golden standard [35]) and an enzymatic cascade that converts creatinine into an end-product for colorimetric or fluorescent read-out $[29,36]$. They both rely on bulky and expensive equipment, such as Pentra C200. Commercially available iSTAT (Abbott) is a handheld amperometric biosensor that uses an enzymatic cascade to detect creatinine in whole blood, and is considered a POC device, although it has high cost $(\sim \$ 14000)$.

\begin{tabular}{lllll}
\hline & Pentra C200 & Reflotron & i-STAT system & Creasensor \\
\hline Readout & Colorimetric & Dye reflectance & Amperometric & Colorimetric \\
Reaction & Jaffé & Enzymatic & Enzymatic & Enzymatic \\
Sample type & Serum, plasma & Whole blood & Whole blood & Plasma \\
Sample volume & $9 \mu \mathrm{L}$ & $30 \mu \mathrm{L}$ & $65 \mu \mathrm{L}$ & $5 \mu \mathrm{l}$ \\
Time-to-result & $510 \mathrm{~s}$ & $120 \mathrm{~s}$ & $120 \mathrm{~s}$ & $300 \mathrm{~s}$ \\
Measurement range & $0.14-18 \mathrm{mg} / \mathrm{dL}$ & $0.50-10 \mathrm{mg} / \mathrm{dL}$ & $0.20-20 \mathrm{mg} / \mathrm{dL}$ & $0.67-20 \mathrm{mg} / \mathrm{dL}$ \\
Operating temperature & $\mathrm{NA}$ & $37^{\circ} \mathrm{C}$ & $37^{\circ} \mathrm{C}$ & $37^{\circ} \mathrm{C}$ \\
\cline { 2 - 5 } & & &
\end{tabular}




$\begin{array}{llll}\sim \$ 37000 & \sim \$ 9000 & \sim \$ 14000 & \sim \$ 300^{*} \\ \sim \$ 25 & \sim \$ 1.5 & \sim \$ 14.5 & \sim \$ 5^{*} \\ \text { Benchtop (95 kg) } & \text { Benchtop }(5 \mathrm{~kg}) & \text { Handheld }(0.7 \mathrm{~kg}) & \text { Benchtop }(<1 \mathrm{~kg})\end{array}$

*These values represent an estimated cost of the products

368 In particular, the SIMPLE platform works in withdrawing mode, thus pulling liquids into

369 a microfluidic network while offering a wide range of flow rates. No external power is needed and the SIMPLE system is robust, easy to fabricate and easy to use, inexpensive, and suited for mass replication technologies, addressing most of the POC requirements. If compared to paperbased microfluidic devices, the sample introduced in the SIMPLE-based cartridge does not get in contact with the porous material avoiding matrix interferences. Moreover, having the detection zone in an optically transparent channel allows for different detection methods to be used (i.e. colorimetric, fluorescence, chemiluminescence). Finally, the flexibility of the SIMPLE concept towards different sample matrices and liquid volumes combined with unique advantages of Creasensor device (such as real-time monitoring of the assay reaction, ease-of-use, portability and low cost) make it a very attractive POC technology for other applications.

\section{Conclusions}

In this paper, we presented a fully integrated SIMPLE-based biosensor, named Creasensor, engineered to detect creatinine in blood plasma samples. The Creasensor combines fast bioassay on a self-powered SIMPLE microfluidic cartridge (Creacard) with integrated signal processing unit and a simple but robust colorimetric read-out. The Creacard was designed to provide efficient sample manipulation and optimal conditions for the read-out system. The enzymatic bioassay was optimized to (i) meet POC requirements by significantly decreasing the TTR (from 60 to $5 \mathrm{~min}$ ) and the sample volume (from 50 to $5 \mu \mathrm{L}$ ) compared to the commercially available enzymatic kit, (ii) cover clinically relevant concentration range of creatinine (i.e. 0.76 $20 \mathrm{mg} / \mathrm{dL}$ ) and (iii) be applied in plasma matrices. Finally, the Creasensor was validated on field in a single-blind study by measuring creatinine levels in 16 creatinine-spiked plasma samples, showing excellent agreement between the measured values and the spiked concentrations, as confirmed with an ICC of 0.97. The developed Creasensor demonstrated equivalent analytical performance to the golden standards in this field while offering several advantages, such as real-time monitoring of the assay reaction, ease-of-use, portability and lower cost over many commercial devices (as indicated in Table 1 )

In conclusion, this study showed for the first time that a SIMPLE-based microfluidic cartridge can completely support the implementation of relevant biological assay. Since the Creacard is flexible in terms of liquid manipulation and compatible with different bioassay strategies (i.e. ELISA) and sample matrices (i.e. urine, saliva), one can envision that bioassays for other relevant biomarkers, such as cholesterol, albumin, troponin and CRP, could easily be integrated on this innovative microfluidic platform expanding its portfolio of POC applications. In fact, different combinations of multiple pumps are under development to allow sample preparation on chip, complex multi-step protocols and different read-out approaches, all in a self-powered, portable and cost effective manner. Finally, clinical studies with real patients' samples will be performed in the future to confirm the SIMPLE platform as valid alternative in the POCT field. 
410 The research leading to these results has received funding from the KU Leuven (PDM/17/093, 411 C3 project 3E150475, C3 project C32/17/007) and the FWO-Flanders Postdoctoral Fellow Devin Daems 12U1618N. These results were obtained in the context of the SensUs 2016 competition (http://sensus.org/).

414

\section{References}

[1] Y. Temiz, R.D. Lovchik, G. V. Kaigala, E. Delamarche, Lab-on-a-chip devices: how to close and plug the lab?, Microelectron. Eng. 132 (2014) 156-175. doi:10.1016/j.mee.2014.10.013.

[2] C.D. Chin, V. Linder, S.K. Sia, Commercialization of microfluidic point-of-care diagnostic devices., Lab Chip. 12 (2012) 2118-34. doi:10.1039/c2lc21204h.

[3] M.I. Mohammed, S. Haswell, I. Gibson, Lab-on-a-chip or Chip-in-a-lab: Challenges of Commercialization Lost in Translation, Procedia Technol. 20 (2015) 54-59. doi:10.1016/j.protcy.2015.07.010.

[4] L. Gervais, N. de Rooij, E. Delamarche, Microfluidic Chips for Point-of-Care Immunodiagnostics, Adv. Mater. 23 (2011) H151-H176. doi:10.1002/adma.201100464.

[5] L.R. Volpatti, A.K. Yetisen, Commercialization of microfluidic devices., Trends Biotechnol. 32 (2014) 347-50. doi:10.1016/j.tibtech.2014.04.010.

[6] E.K. Sackmann, A.L. Fulton, D.J. Beebe, The present and future role of microfluidics in biomedical research., Nature. 507 (2014) 181-9. doi:10.1038/nature13118.

[7] G.M. Whitesides, Cool, or simple and cheap? Why not both?, Lab Chip. 13 (2013) 11-13. doi:10.1039/C2LC90109A.

[8] R. Safavieh, D. Juncker, Capillarics: pre-programmed, self-powered microfluidic circuits built from capillary elements., Lab Chip. 13 (2013) 4180-9. doi:10.1039/c3lc50691f.

[9] D.Y. Liang, A.M. Tentori, I.K. Dimov, L.P. Lee, Systematic characterization of degas-driven flow for poly(dimethylsiloxane) microfluidic devices., Biomicrofluidics. 5 (2011) 24108. doi:10.1063/1.3584003.

[10] I.K. Dimov, L. Basabe-Desmonts, J.L. Garcia-Cordero, B.M. Ross, Y. Park, A.J. Ricco, L.P. Lee, Stand-alone self-powered integrated microfluidic blood analysis system (SIMBAS)., Lab Chip. 11 (2011) 845-50. doi:10.1039/c0lc00403k.

[11] G. Li, Y. Luo, Q. Chen, L. Liao, J. Zhao, A "place n play" modular pump for portable microfluidic applications., Biomicrofluidics. 6 (2012) 14118-1411816. doi:10.1063/1.3692770.

[12] K. Hosokawa, K. Sato, N. Ichikawa, M. Maeda, Power-free poly(dimethylsiloxane) microfluidic devices for gold nanoparticle-based DNA analysis., Lab Chip. 4 (2004) 1815. doi:10.1039/b403930k.

[13] K.W. Oh, C.H. Ahn, A review of microvalves, J . Micromech . Microeng. 16 (2006) 13-39. doi:10.1088/0960-1317/16/5/R01.

[14] A.W. Martinez, S.T. Phillips, G.M. Whitesides, Three-dimensional microfluidic devices fabricated in layered paper and tape., Proc. Natl. Acad. Sci. U. S. A. 105 (2008) 19606-11. doi:10.1073/pnas.0810903105.

[15] D.M. Cate, J.A. Adkins, J. Mettakoonpitak, C.S. Henry, Recent developments in paper-based microfluidic devices., Anal. Chem. 87 (2015) 19-41. doi:10.1021/ac503968p.

[16] A.W. Martinez, S.T. Phillips, G.M. Whitesides, E. Carrilho, Diagnostics for the developing world: microfluidic paper-based analytical devices., Anal. Chem. 82 (2010) 3-10. doi:10.1021/ac9013989.

[17] X. Li, D.R. Ballerini, W. Shen, A perspective on paper-based microfluidics: Current status and future trends., Biomicrofluidics. 6 (2012) 11301-1130113. doi:10.1063/1.3687398.

[18] T. Kokalj, Y. Park, M. Vencelj, M. Jenko, L.P. Lee, Self-powered Imbibing Microfluidic Pump by Liquid Encapsulation: SIMPLE., Lab Chip. 14 (2014) 4329-33. 
doi:10.1039/c4lc00920g.

[19] A.S. Levey, L.A. Stevens, C.H. Schmid, Y.L. Zhang, A.F. Castro, H.I. Feldman, J.W. Kusek, P. Eggers, F. Van Lente, T. Greene, J. Coresh, CKD-EPI (Chronic Kidney Disease Epidemiology Collaboration), A new equation to estimate glomerular filtration rate., Ann. Intern. Med. 150 (2009) 604-12. http://www.ncbi.nlm.nih.gov/pubmed/19414839 (accessed February 9, 2017).

[20] C.S. Pundir, S. Yadav, A. Kumar, Creatinine sensors, TrAC - Trends Anal. Chem. 50 (2013) 42-52. doi:10.1016/j.trac.2013.04.013.

[21] O.J. Wouters, D.J. O’Donoghue, J. Ritchie, P.G. Kanavos, A.S. Narva, Early chronic kidney disease: diagnosis, management and models of care, Nat. Rev. Nephrol. 11 (2015) 491502. doi:10.1038/nrneph.2015.85.

[22] M. Shephard, M. Peake, O. Corso, A. Shephard, B. Mazzachi, B. Spaeth, J. Barbara, T. Mathew, Assessment of the Nova StatSensor whole blood point-of-care creatinine analyzer for the measurement of kidney function in screening for chronic kidney disease, Clin. Chem. Lab. Med. 48 (2010) 1113-1119. doi:10.1515/CCLM.2010.238.

[23] P.K. Yuen, V.N. Goral, Low-cost rapid prototyping of flexible microfluidic devices using a desktop digital craft cutter., Lab Chip. 10 (2010) 384-7. doi:10.1039/b918089c.

[24] F. Dal Dosso, T. Kokalj, D. Spasic, J. Lammertyn, Self-powered programmable microfluidic platform for LOC applications, in: Proc. 20th Int. Conf. Miniaturized Syst. Chem. Life Sci. $\mu$ TAS, Dublin, Ireland, 2016: pp. 990-991.

[25] G.G. Kock, Intraclass correlation coefficient, John Wiley and Sons, New York, 1982.

[26] S.K. Vashist, P.B. Luppa, L.Y. Yeo, A. Ozcan, J.H.T.T. Luong, Emerging Technologies for Next-Generation Point-of-Care Testing., Trends Biotechnol. 33 (2015) 692-705. doi:10.1016/j.tibtech.2015.09.001.

[27] A. St John, C.P. Price, Existing and Emerging Technologies for Point-of-Care Testing., Clin. Biochem. Rev. 35 (2014) 155-67. http://www.ncbi.nlm.nih.gov/pubmed/25336761 (accessed May 6, 2016).

[28] W. Junge, B. Wilke, A. Halabi, G. Klein, Determination of reference intervals for serum creatinine, creatinine excretion and creatinine clearance with an enzymatic and a modified Jaffé method, Clin. Chim. Acta. 344 (2004) 137-148. doi:10.1016/j.cccn.2004.02.007.

[29] A.J. Killard, M.R. Smyth, Creatinine biosensors: principles and designs, Trends Biotechnol. 18 (2000) 433-437. doi:10.1016/S0167-7799(00)01491-8.

[30] T. Guinovart, D. Hernández-Alonso, L. Adriaenssens, P. Blondeau, F.X. Rius, P. Ballester, F.J. Andrade, Characterization of a new ionophore-based ion-selective electrode for the potentiometric determination of creatinine in urine, Biosens. Bioelectron. 87 (2017) 587-592. doi:10.1016/j.bios.2016.08.025.

[31] Jae Ho Shin, Yong Suk Choi, Han Jin Lee, Sung Hyuk Choi, Jeonghan Ha, In Jun Yoon, and Hakhyun Nam, G.S. Cha*, A Planar Amperometric Creatinine Biosensor Employing an Insoluble Oxidizing Agent for Removing Redox-Active Interferences, (2001). doi:10.1021/AC010497A.

[32] K. Talalak, J. Noiphung, T. Songjaroen, O. Chailapakul, W. Laiwattanapaisal, A facile lowcost enzymatic paper-based assay for the determination of urine creatinine, Talanta. 144 (2015) 915-921. doi:10.1016/j.talanta.2015.07.040.

[33] O. Gbinigie, M. Thompson, C.P. Price, C. Heneghan, A. Plüddemann, Point-of-Care creatinine testing for the detection and monitoring of chronic kidney disease, 2014. https://www.oxford.dec.nihr.ac.uk/reports-and-resources/horizon-scanningreports/point-of-care-creatinine-testing-for-the-detection-and-monitoring-of-chronickidney-disease (accessed February 10, 2017).

[34] M.D. Shephard, Point-of-Care Testing and Creatinine Measurement., Clin. Biochem. Rev. 32 (2011) 109-14. http://www.ncbi.nlm.nih.gov/pubmed/21611085 (accessed February 9, 2017).

[35] L. Hoste, K. Deiteren, H. Pottel, N. Callewaert, F. Martens, Routine serum creatinine measurements: how well do we perform?, BMC Nephrol. 16 (2015) 21. 
doi:10.1186/s12882-015-0012-x.

[36] J.R. Delanghe, M.M. Speeckaert, Creatinine determination according to Jaffe--what does it stand for?, Clin. Kidney J. 4 (2011) 83-86. doi:10.1093/ndtplus/sfq211. 